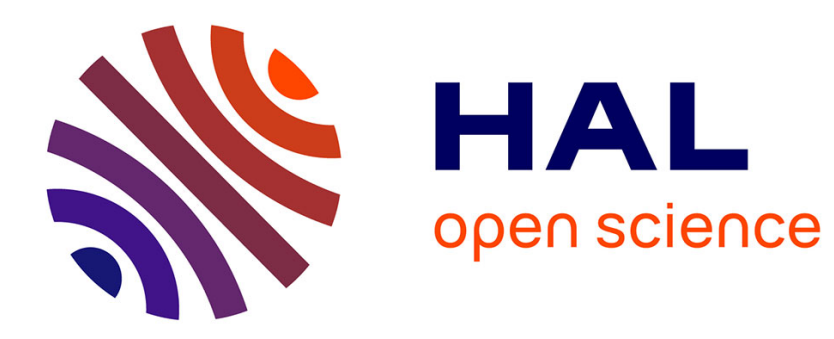

\title{
Rate of decay to 0 of the solutions to a nonlinear parabolic equation
}

Imen Ben Arbi

\section{To cite this version:}

Imen Ben Arbi. Rate of decay to 0 of the solutions to a nonlinear parabolic equation. 2010. hal00517387

\author{
HAL Id: hal-00517387 \\ https://hal.science/hal-00517387
}

Preprint submitted on 14 Sep 2010

HAL is a multi-disciplinary open access archive for the deposit and dissemination of scientific research documents, whether they are published or not. The documents may come from teaching and research institutions in France or abroad, or from public or private research centers.
L'archive ouverte pluridisciplinaire HAL, est destinée au dépôt et à la diffusion de documents scientifiques de niveau recherche, publiés ou non, émanant des établissements d'enseignement et de recherche français ou étrangers, des laboratoires publics ou privés. 


\title{
Rate of decay to 0 of the solutions to a nonlinear parabolic equation
}

\author{
I.Ben $\mathrm{Arbi}^{1}$
}

Résumé: On étudie l'ordre de convergence vers 0 , quand $t \longrightarrow+\infty$ de la solution de l'équation $\psi_{t}-\Delta \psi+|\psi|^{p-1} \psi=0$ avec les conditions aux limites de Neumann dans un ouvert connexe borné de $\mathbb{R}^{n}$ où $p>1$. On montre que soit $\psi(t, \cdot)$ converge vers 0 exponentiellement, soit $\psi(t, \cdot)$ décroît comme $t^{-\frac{1}{(p-1)}}$.

Abstract: We study the decay rate to 0 , as $t \longrightarrow+\infty$ of the solution of equation $\psi_{t}-\Delta \psi+|\psi|^{p-1} \psi=0$ with Neumann boundary conditions in a bounded smooth open connected domain of $\mathbb{R}^{n}$ where $p>1$. We show that either $\psi(t, \cdot)$ converges to 0 exponentially fast or $\psi(t, \cdot)$ decreases like $t^{-\frac{1}{(p-1)}}$.

Keywords: rate of decay, parabolic equation

\footnotetext{
${ }^{1}$ Laboratoire Jacques-Louis Lions, Université Pierre et Marie Curie - Paris 6, boîte courrier 187, 4 place Jussieu, 75252 Paris Cedex 05, FRANCE.
} 


\section{Introduction and main results.}

In this paper we consider the following nonlinear parabolic equation

$$
\left\{\begin{array}{l}
\psi_{t}-\Delta \psi+g(\psi)=0 \quad \text { in } \quad \mathbb{R}^{+} \times \Omega \\
\frac{\partial \psi}{\partial n}=0 \quad \text { on } \quad \mathbb{R}^{+} \times \partial \Omega
\end{array}\right.
$$

where $\Omega$ is a bounded smooth open connected domain of $\mathbb{R}^{n}$ and $g \in C^{1}(\mathbb{R})$ satisfies

$$
g(0)=0
$$

and for some $p>1$

$$
\exists c>0, \forall s \in \mathbb{R}, \quad 0 \leq g^{\prime}(s) \leq c|s|^{p-1} .
$$

From (1.2)-(1.3)we deduce that $g(s)$ has the sign of $s$ and

$$
\forall s \in \mathbb{R}, \quad|g(s)| \leq \frac{c}{p}|s|^{p} .
$$

We define the operator $A$ by

$$
D(A)=\left\{\psi \in H^{2}(\Omega), \frac{\partial \psi}{\partial n}=0 \quad \text { on } \quad \partial \Omega\right\}
$$

and

$$
\forall \psi \in D(A), \quad A \psi=-\Delta \psi
$$

On the other hand the operator $B$ defined by

$$
D(B)=\left\{\psi \in L^{2}(\Omega),-\Delta \psi+g(\psi) \in L^{2}(\Omega) \text { and } \frac{\partial \psi}{\partial n}=0 \quad \text { on } \quad \partial \Omega\right\}
$$

and

$$
\forall \psi \in D(B), \quad B \psi=-\Delta \psi+g(\psi)
$$

is well-known to be maximal monotone in $L^{2}(\Omega)$. As a consequence of $[1,2]$ for any $\psi_{0} \in L^{2}(\Omega)$ there exists a unique weak solution of the equation

$$
\psi^{\prime}+B \psi=0 \quad \text { on } \quad \mathbb{R}^{+} ; \quad \psi(0, x)=\psi_{0} .
$$

In addition it is well known that if $\psi_{0} \in L^{\infty}(\Omega), \psi(t, \cdot)$ remains in $L^{\infty}(\Omega)$ for all $t>0$. Finally [8] contains an estimate of the solution in $C(\bar{\Omega})$ and $C^{1}(\bar{\Omega})$ for $t>0$, which is valid for any sufficiently regular domain. 
Concerning the behaviour for $t$ large, in [5], A.Haraux established in the case of a pure power nonlinearity the exponential convergence to 0 of the projection on the range of $A$ of the solution of equation (1.1). Moreover in [4], the study of the equation $u^{\prime \prime}+u^{\prime}-\Delta u+g(u)=0$ with Neumann boundary conditions and where $g$ satisfies $\exists C, c>0, \forall s \in \mathbb{R}, c|s|^{p-1} \leq g^{\prime}(s) \leq C|s|^{p-1}$ for some $p>1$, showed that either $u(t)$ converges to 0 exponentially fast, or $\|u(t)\|_{H_{0}^{1}(\Omega)} \geq \gamma t^{-1 /(p-1)}$ with $\gamma>1$ for $t \geq 1$.

Several authors have treated some variants of equation (1.1), for example in [6] with $g(u)=c|u|^{p-1} u-\lambda_{1} u$ and with Dirichlet boundary conditions, was studied the decay rate at the infinity of solutions to (1.1), where $\lambda_{1}>0$ is the first eigenvalue of $-\Delta$ in $H_{0}^{1}(\Omega)$. The result obtained there is optimal for positive solutions.

According to La Salle's invariance principle, cf. [3, 7], any solution $\psi$ of (1.1), having a precompact range on $\mathbb{R}^{+}$with values to $L^{\infty}(\Omega)$, converges to a continuum of stationary solutions of equation (1.1), which reduces here to the constants of some sub-interval of $g^{-1}(0)$. By monotonicity, it is in fact known that $\psi$ converges to some constant $a \in g^{-1}(0)$.

Our first result is valid without any additional hypothesis on $g$

Theorem 1.1. Let g satisfy (1.2) and (1.3). Then any solution $\psi$ of (1.1) satisfies the following alternative as $t \rightarrow \infty$ : either

$$
\|\psi(t, \cdot)\|_{\infty} \leq C e^{-\lambda_{2} t}
$$

or

$$
\exists c^{\prime}>0, \forall t \geq 1, \quad\left|\int_{\Omega} \psi(t, x) d x\right| \geq c^{\prime} t^{-\frac{1}{p-1}},
$$

where $\lambda_{2}>0$ is the second eigenvalue of $A$ in $D(A)$.

Our second result provides a more accurate estimate when $g(\psi)=|\psi|^{p-1} \psi$

Theorem 1.2. Let us consider the nonlinear parabolic problem (1.1) with $g(\psi)=|\psi|^{p-1} \psi$, then any solution $\psi$ of (1.1) satisfies the following alternative as $t \rightarrow \infty$ : either

$$
\|\psi(t, \cdot)\|_{\infty} \leq C e^{-\lambda_{2} t}
$$

or

$$
\forall t \geq 1, \quad|||\psi(t, \cdot)|-((p-1) t)^{-\frac{1}{p-1}} \|_{\infty} \leq K t^{-\frac{1}{(p-1)}-1},
$$

where $K, C>0, p>1$ and $\lambda_{2}>0$ is the second eigenvalue of $A$ in $D(A)$. 
In the following Proposition, we consider two special cases showing that both possibilities in the second result in the Theorem 1.1 can actually happen.

Proposition 1.3. Let g satisfy (1.2) and (1.3). Then we have

(i) If $\Omega$ is symmetric around $0, g$ is odd and $\psi(0, \cdot)$ is an odd function in $\Omega$, then any solution of (1.1) satisfies (1.6).

(ii) If $\psi(0, \cdot) \geq 0$ and $\psi$ does not vanish a.e in $\Omega$, then any solution of (1.1) satisfies (1.7).

Finally, our last result shows that the second possibility is sharp for a class of functions $g$ more general than the pure power

Proposition 1.4. Under the additional hypothesis

$$
\exists k_{1}>0, \forall s \in \mathbb{R},|g(s)| \geq k_{1}|s|^{p}
$$

for any solution $\psi$ of (1.1), we have

$$
\forall t \geq 1, \quad\|\psi(t, \cdot)\|_{\infty} \leq\left\{\frac{1}{k_{1}(p-1)}\right\}^{\frac{1}{p-1}} t^{-\frac{1}{p-1}}
$$

\section{Proof of Proposition 1.4.}

Proof. If $\psi(0, \cdot)=0$, we have $\psi(t, \cdot) \equiv 0$ and the result is obvious. Otherwise let $z$ be defined by

$$
z(t)=\left\{\frac{1}{\|\psi(0, \cdot)\|_{\infty}^{1-p}+k_{1}(p-1) t}\right\}^{\frac{1}{p-1}}
$$

Then $z$ is a solution of the following nonlinear ODE problem

$$
\left\{\begin{array}{l}
z^{\prime}+k_{1} z^{p}=0 \\
z(0)=\|\psi(0, \cdot)\|_{\infty}
\end{array}\right.
$$

Under the additional condition(1.10), we will show that $z$ is a super solution of (1.1). Indeed, we have

$$
\begin{aligned}
z_{t}-\Delta z+g(z) & =-k_{1}\left(z(0)^{1-p}+k_{1}(p-1) t\right)^{-\frac{p}{p-1}}+g(z) \\
& \geq-k_{1}\left(z(0)^{1-p}+k_{1}(p-1) t\right)^{-\frac{p}{p-1}}+k_{1}|z|^{p} \\
& \geq 0 .
\end{aligned}
$$


Since $\psi(0, \cdot) \leq z(0)$ we deduce, by standard comparison principle, that $\psi(t, \cdot) \leq z(t) \forall t \geq 1$.

A similar calculation shows that $\psi(t, \cdot) \geq-z(t), \quad \forall t \geq 1$ which concludes the proof.

\section{A general result on the range component.}

Defining the orthogonal projection $P: H \longrightarrow N$, where

$$
H=L^{2}(\Omega), N=\operatorname{ker}(A) \text { and } P \psi(t, \cdot)=\frac{1}{|\Omega|} \int_{\Omega} \psi(t, x) d x,
$$

as already mentioned in the introduction, [5] showed that for $g(\psi)=|\psi|^{p-1} \psi$, the following estimate holds

$$
\|\psi(t)-P \psi(t)\|_{L^{2}(\Omega)} \leq K e^{-\lambda_{2} t},
$$

for some constant $K>0$. In this section, we will show that we have the same result for any function $g$ satisfying (1.3).

Proposition 3.1. Let $\psi \in C\left(\mathbb{R}^{+}, L^{\infty}\right)$ be any solution of (1.1). Assume that $g$ is a locally Lipschitz non-decreasing function. Then we have,

$$
\|\psi(t)-P \psi(t)\|_{2} \leq\|\psi(0)-P \psi(0)\|_{2} e^{-\lambda_{2} t}
$$

where $\|.\|_{2}$ denotes the norm in $L^{2}(\Omega)$ and $\lambda_{2}>0$ is the second eigenvalue of $-\Delta$ in $L^{2}(\Omega)$ with Neumann boundary conditions .

Proof. We denote by $(u, v)$ the inner product of two functions $u, v$ of $L^{2}(\Omega)$. Since $g$ is a nondecreasing function, for all $\psi \in L^{\infty}(\Omega)$, we have a.e. in $x \in \Omega$

$$
(g(\psi)-g(P \psi))(\psi-P \psi) \geq 0
$$

and then by integrating over $\Omega$

$$
(g(\psi), \psi-P \psi)-(g(P \psi), \psi-P \psi) \geq 0 .
$$

Since $g(P \psi)$ is a constant and $(\psi-P \psi) \in N^{\perp}$, we deduce that $(g(P \psi), \psi-$ $P \psi)=0$. Hence from (3.2),

$$
(g(\psi), \psi-P \psi) \geq 0 .
$$




\section{Setting}

$$
w=\psi-P \psi,
$$

we have since $\Delta P \psi=P \Delta \psi=0$

$$
w^{\prime}-\Delta w=\psi^{\prime}-\Delta \psi-P \psi^{\prime}+\Delta P \psi=(I-P)\left(\psi^{\prime}-\Delta \psi\right)=-(I-P) g(\psi) \text {. }
$$

Thus, since $(w,(I-P) g(\psi))=((I-P) w, g(\psi))=(w, g(\psi))=(g(\psi), \psi-P \psi)$ we find

$$
\frac{1}{2} \frac{d}{d t}\|w(t)\|_{2}^{2}=(w, \Delta w)-(g(\psi), \psi-P \psi) \leq-\lambda_{2}|w|^{2} .
$$

By integrating we obtain (3.1).

\section{Proof of Theorem 1.1.}

We set $\psi=u+w$, where $u=P \psi$ and $w=(I-P) \psi$. By projecting (1.1) on $N$ we obtain

$$
u^{\prime}+P(g(\psi))=0,
$$

where we have used that $P(A \psi)=0$, since $R(A) \subset N^{\perp}$. Noticing that

$$
u^{\prime}+P(g(u))+P(g(\psi)-g(u))=u^{\prime}+g(u)+P(g(\psi)-g(u)),
$$

we can rewrite the equation (4.1) as

$$
u^{\prime}+g(u)=-P(g(\psi)-g(u)) .
$$

By the assumption (1.3), we deduce that

$$
|P(g(\psi)-g(u))| \leq \frac{1}{|\Omega|}\|g(\psi)-g(u)\|_{1} \leq \frac{c}{|\Omega|}\left(\|\psi\|_{2 p-2}^{p-1}+\|u\|_{2 p-2}^{p-1}\right)\|w\|_{2} .
$$

But $\psi$ and $u$ are uniformly bounded and from Proposition 3.1 we have the estimate $\|w(t)\|_{2} \leq K e^{-\lambda_{2} t}$. Therefore

$$
|P(g(\psi)-g(u))| \leq K^{\prime} e^{-\lambda_{2} t},
$$

with $K^{\prime}>0$. Which leads us to study the equation:

$$
u^{\prime}+g(u)=f(t) \quad \text { in } \quad \mathbb{R}^{+},
$$

where

$$
f(t)=P(g(\psi)-g(u))
$$

and

$$
|f(t)| \leq K^{\prime} e^{-\lambda_{2} t}
$$

Using the same method as in [5], we show the following result: 
Lemma 4.1. Let $c>0, \gamma>0, p>1$ and $g$ satisfying (1.2) and (1.3) Let $M>0$ such that

$$
M \leq\left(\frac{\gamma}{2 c}\right)^{\frac{1}{p-1}}
$$

$c_{1}>0$ with

$$
c_{1} \leq \frac{\gamma}{2} M,
$$

then for every function $f$ satisfying

$$
|f(t)| \leq c_{1} e^{-\gamma t}
$$

there exists a unique function $v \in C^{1}\left(\mathbb{R}^{+}\right)$satisfying

$$
\forall t \geq 0, \quad v^{\prime}+g(v)=f(t)
$$

and

$$
\sup _{t \in(0,+\infty)}\left\{e^{\gamma t}|v(t)|\right\} \leq M
$$

Proof. Since any solution of (4.4).(4.5) satisfies the integral equation

$$
v(t)=-\int_{t}^{+\infty}(f(s)-g(v(s)) d s .
$$

We look for a solution of (4.6). It is then natural to introduce the following function space :

$$
X=\left\{v \in C(0,+\infty) ; \sup _{t \in(0,+\infty)} e^{\gamma t}|v(t)| \leq M\right\}
$$

equipped with the distance associated to the norm

$$
\|v\|_{\gamma}=\sup _{t \in(0,+\infty)} e^{\gamma t}|v(t)|
$$

We consider the operator $\mathcal{T}: X \rightarrow C(0,+\infty)$ defined by

$$
\mathcal{T} v(t)=-\int_{t}^{+\infty}(f(s)-g(v(s)) d s
$$

From (1.4), we have the estimate

$$
\forall s \in \mathbb{R}^{+} ; \quad|g(v(s))| \leq \frac{c}{p}|v(s)|^{p} .
$$


First we will show that $\mathcal{T}(X) \subset X$. Let $v \in X$, then for all $t \geq 0$,

$$
\begin{aligned}
|\mathcal{T} v(t)| & \leq \int_{t}^{+\infty}|f(s)| d s+\int_{t}^{+\infty}|g(v(s))| d s \\
& \leq \int_{t}^{+\infty}|f(s)| d s+\frac{c}{p} \int_{t}^{+\infty}|v(s)|^{p} d s \\
& \leq \frac{c_{1}}{\gamma} e^{-\gamma t}+\frac{c}{p} M^{p} \int_{t}^{+\infty} e^{-p \gamma s} d s \\
& \leq\left(\frac{c_{1}}{\gamma}+\frac{c M^{p}}{p^{2} \gamma}\right) e^{-\gamma t} \\
& \leq\left(\frac{M}{2}+M c \frac{M^{p-1}}{p^{2} \gamma}\right) e^{-\gamma t} \\
& \leq\left(\frac{M}{2}+\frac{M}{2 p^{2}}\right) e^{-\gamma t} .
\end{aligned}
$$

Since $p>1$, it follows that

$$
|\mathcal{T} v(t)| \leq M e^{-\gamma t}
$$

Hence by (4.5), we obtain that $\mathcal{T} v \in X$, with

$$
\|\mathcal{T} v(t)\|_{\gamma} \leq M
$$

Secondly, we will prove that $\mathcal{T}$ is a contraction on $X$. In fact, for $x, \bar{x} \in X$ and for all $t \geq 0$

$$
\begin{aligned}
|\mathcal{T} x(t)-\mathcal{T} \bar{x}(t)| & \leq \int_{t}^{+\infty}|g(x(s))-g(\bar{x}(s))| d s \\
& \leq c M^{p-1} \int_{t}^{+\infty} e^{-p \gamma s} e^{\gamma s}|x(s)-\bar{x}(s)| d s \\
& \leq \frac{c M^{p-1}}{p \gamma}\|x-\bar{x}\|_{\gamma} e^{-\gamma t} .
\end{aligned}
$$

Then we have

$$
|\mathcal{T} x(t)-\mathcal{T} \bar{x}(t)| e^{\gamma t} \leq \frac{c M^{p-1}}{p \gamma}\|x-\bar{x}\|_{\gamma} .
$$

Therefore, since $M^{p-1}$ satisfies (4.3), we conclude that $\forall x, \bar{x} \in X$

$$
\|\mathcal{T} x-\mathcal{T} \bar{x}\|_{\gamma} \leq \frac{1}{2}\|x-\bar{x}\|_{\gamma}
$$


Thus $\mathcal{T}$ is a $\frac{1}{2}$-Lipschitz functional on the complete metric space $X$ and the result follows from the Banach fixed point theorem. From (4.6) it follows easily that $v$ satisfies (4.4). Then the uniqueness of $v$ follows from the uniqueness of the solution of (4.6) ( $\mathcal{T}$ is a contraction) and the fact that any solution of (4.4) satisfies (4.6). The existence comes from the fact that conversely any solution of (4.6) satisfies (4.4).

Proof of Theorem 1.1 continued. Consequently, we have a solution $v$ that satisfies the equation (4.4) and for all $t \geq T_{0}$

$$
|v(t)| \leq M e^{-\lambda_{2} t}
$$

where $M=M^{\prime} e^{\gamma T_{0}}$ and $M^{\prime}>0$. If we subtract (4.4) from (4.2) we obtain

$$
(u-v)^{\prime}+g(u)-g(v)=0
$$

Setting $z=u-v$, we complete the proof analyzing two cases.

$\underline{1^{\text {st }} \text { case: }}$ If $z\left(T_{0}\right)=0$, then for all $t \geq T_{0}, z(t)=0$. Hence $u \equiv v$ and from (4.8) it follows that

$$
|u(t)| \leq M e^{-\lambda_{2} t}
$$

Then, using (3.1), we obtain

$$
\|\psi(t)\|_{2} \leq M^{\prime} e^{-\lambda_{2} t}
$$

Finally by reasoning as in [5], [6] we obtain (1.6).

2 ${ }^{\text {nd }}$ case: If $z\left(T_{0}\right) \neq 0$ then $\forall t \geq T_{0}, z(t) \neq 0$ and we have

$$
z^{\prime}(t)+\frac{g(u(t))-g(v(t))}{u(t)-v(t)} z(t)=0 .
$$

Since $g$ is a monotonic function,

$$
\alpha(t):=\frac{g(u(t))-g(v(t))}{u(t)-v(t)}
$$

is a strictly positive function. Moreover, $\exists \theta \in] 0,1[$,

$$
\alpha(t)=g^{\prime}(\theta u(t)+(1-\theta) v(t)) \leq c|\theta u(t)+(1-\theta) v(t)|^{p-1}
$$

We distinguish 2 cases:

$-p>2$ then by convexity of the $p$ th power we have 
$|\theta u(t)+(1-\theta) v(t)|^{p-1} \leq \theta|u|^{p-1}+(1-\theta)|v|^{p-1} \leq|u|^{p-1}+|v|^{p-1}$.

$-1<p<2$ we study the function $(x+y)^{a}-x^{a}$ for $0<a<1$ and $x, y>0$ we prove that $X \longrightarrow(1+X)^{a}-X^{a}$ is a decreasing function on $(0,+\infty)$, we deduce $0<(1+X)^{a}-X^{a}<1$ and it follows by homogeneity that $(x+y)^{a}<x^{a}+y^{a}$ by letting $X=\frac{x}{y}$. Then we conclude that $\mid \theta u(t)+(1-$ $\theta)\left.v(t)\right|^{p-1} \leq|u|^{p-1}+|v|^{p-1}$. Consequently we obtain

$$
\forall p>1, \quad \alpha(t) \leq c\left(|u|^{p-1}+|v|^{p-1}\right) .
$$

Setting $y=|z|$, we have that

$$
y^{\prime}+\alpha(t) y \geq 0 .
$$

Then the estimate (4.10) implies that

$$
y^{\prime} \geq-c\left(|u|^{p-1}+|v|^{p-1}\right) y \geq-c\left(|z+v|^{p-1}+|v|^{p-1}\right) y .
$$

Hence there exists some constants $c_{2}, c_{3}>0$ such that

$$
y^{\prime} \geq-c_{2}\left(|z|^{p-1}\right) y-c_{3}|v|^{p-1} y .
$$

Since $y=|z|$,

$$
y^{\prime} \geq-c_{2} y^{p}-c_{3}|v|^{p-1} y .
$$

Putting $a(t)=c_{3}|v|^{p-1}$, we deduce that

$$
y^{\prime}+a(t) y \geq-c_{2} y^{p} .
$$

We set

$$
A(t)=-c_{3} \int_{t}^{+\infty}|v|^{p-1} d s, \quad \omega(t)=e^{A(t)} y
$$

and by replacing $\omega$ in (4.11), we obtain

$$
\omega(t) \geq\left\{\frac{1}{\omega(0)^{1-p}+(p-1) c_{4} t}\right\}^{\frac{1}{p-1}}
$$

with $c_{4}=\left(\int_{0}^{+\infty} a(s) d s\right)^{-(p-1)}$. Then for $t$ large enough we have

$$
\omega(t) \geq K t^{-\frac{1}{p-1}}
$$

Since $t \longrightarrow e^{A(t)}$ is a bounded function, we conclude (1.7) by observing that $u=z+v$ and $v$ tends to 0 exponentially at infinity. 


\section{$5 \quad$ Proof of Theorem 1.2.}

Considering $g(\psi)=|\psi|^{p-1} \psi, g$ satisfies (1.2) and (1.3) with $c=p$. Hence Lemma 4.1 is applicable with $c=p$, therefore we assume

$$
M \leq\left(\frac{\lambda_{2}}{2 p}\right)^{\frac{1}{p-1}} .
$$

In (4.2), we replace $g(\psi)=|\psi|^{p-1} \psi$, we can subtract (4.4) from (4.2), we deduce:

$$
(u-v)^{\prime}+|u|^{p-1} u-|v|^{p-1} v=0
$$

We will study two cases.

$1^{\text {st }}$ case: If $z\left(T_{0}\right)=0$ then for all $t \geq T_{0}, z(t)=0$. Hence $u \equiv v$ and from (4.8) it follows that

$$
|u(t)| \leq M e^{-\lambda_{2} t}
$$

Moreover, using (3.1), we obtain (1.8).

$\underline{2^{n d} \text { case: }}$ if $z\left(T_{0}\right) \neq 0$ then for all $t \geq T_{0} z(t) \neq 0$ and we have

$$
z^{\prime}(t)+|u(t)|^{p-1} u(t)-|v(t)|^{p-1} v(t)=z^{\prime}(t)+\alpha(t) z(t)=0 .
$$

with

$$
\begin{aligned}
\alpha(t) & =\frac{|u(t)|^{p-1} u(t)-|v(t)|^{p-1} v(t)}{u(t)-v(t)} \\
& =\frac{|z(t)+v(t)|^{p-1}(z(t)+v(t))-|v(t)|^{p-1} v(t)}{z(t)} \\
& =\frac{|z(t)|^{p-1}\left|1+\frac{v(t)}{z(t)}\right|^{p-1}(z(t)+v(t))-|v(t)|^{p-1} v(t)}{z(t)} .
\end{aligned}
$$

In that case $\alpha(t)>0$, indeed $t \mapsto|u(t)|^{p-1} u(t)$ is non decreasing function. By applying Taylor's formula, we obtain

$$
\alpha(t)=|z(t)|^{p-1}+\beta(t),
$$

with $|\beta(t)| \leq B e^{-\eta t}$, where $\eta>0$ is any positive number smaller than $(p-1) \lambda_{2}$ and $B>0$. Replacing $\alpha$ by its expression in (5.2), equation (5.1) becomes

$$
z^{\prime}+|z|^{p-1} z+\beta(t) z=0 .
$$


Let $y=|z|$, we obtain

$$
y^{\prime}+y^{p}+\beta(t) y=0 .
$$

Setting $\xi(t)=e^{A(t)} y(t)$, with $A(t)=-\int_{t}^{+\infty} \beta(s) d s$ we find

$$
|A(t)| \leq \int_{t}^{+\infty}|\beta(s)| d s \leq \frac{B}{\eta} e^{-\eta t} .
$$

By Taylor's formula we have

$$
\forall h \in[-1,1], \quad\left|e^{h}-1\right| \leq 2|h|,
$$

we can give the following estimate

$$
|\xi(t)-y(t)| \leq y(t)\left|e^{A(t)}-1\right| \leq 2|A(t)| y(t) \leq \frac{2 B}{\eta}\|y\|_{\infty} e^{-\eta t},
$$

we conclude

$$
|\xi(t)-y(t)| \leq k e^{-\delta t},
$$

where $\delta=\eta$ and $k=\frac{2 B}{\eta}\|y\|_{\infty}$.

Replacing $\xi(t)$ in (5.3), we have

$$
\begin{aligned}
& -e^{-A(t)} \xi^{\prime}(t)=e^{-p A(t)} \xi^{p}(t) \\
& \Leftrightarrow-\frac{\xi^{\prime}(t)}{\xi^{p}(t)}=e^{(1-p) A(t)} \\
& \Leftrightarrow\left(\frac{1}{\xi^{p-1}(t)}\right)^{\prime}=(p-1) e^{(1-p) A(t)} .
\end{aligned}
$$

$t \mapsto e^{-(p-1) A(t)}$ is bounded and tends to 1 at infinity, $\xi$ is given by

$$
\xi(t)^{p-1}=\frac{1}{\xi\left(t_{0}\right)^{1-p}+(p-1) \int_{t_{0}}^{t} e^{-(p-1) A(s)} d s} .
$$

we set

$$
D(t)=\xi\left(t_{0}\right)^{1-p}+(p-1) \int_{t_{0}}^{t} e^{-(p-1) A(s)} d s \quad \text { and } \quad h(t)=-(p-1) A(t),
$$

then we show that $D(t)-(p-1) t$ is bounded.

From (5.5), we know that $t \mapsto h(t)$ is an integrable function, then by (5.6), $\left(e^{-(p-1) A(t)}-1\right)$ is also integrable. In order to show (1.9), we proceed 
as follows

$$
\begin{aligned}
|D(t)-(p-1) t| & =\left|\xi\left(t_{0}\right)^{1-p}+(p-1) \int_{t_{0}}^{t} e^{-(p-1) A(s)} d s-(p-1) t\right| \\
& =\left|\xi\left(t_{0}\right)^{1-p}+(p-1) \int_{t_{0}}^{t}\left(e^{-(p-1) A(s)}-1\right) d s-(p-1) t_{0}\right|
\end{aligned}
$$

Using (5.6) we obtain

$$
\begin{aligned}
|D(t)-(p-1) t| & \leq|K|+2(p-1)^{2} \int_{t_{0}}^{t}|A(s)| d s \\
& \leq M_{1}=|K|+2(p-1)^{2} \frac{B}{\eta^{2}}
\end{aligned}
$$

with $K=\xi\left(t_{0}\right)^{1-p}-(p-1) t_{0}$. Setting $d(t)=D(t)-(p-1) t$, we obtain

$$
\begin{aligned}
\left|\xi(t)-\left(\frac{1}{(p-1) t}\right)^{\frac{1}{(p-1)}}\right| & =\left|\left(\frac{1}{D(t)}\right)^{\frac{1}{(p-1)}}-\left(\frac{1}{(p-1) t}\right)^{\frac{1}{(p-1)}}\right| \\
& =\left|\left(\frac{1}{D(t)-(p-1) t+(p-1) t}\right)^{\frac{1}{(p-1)}}-\left(\frac{1}{(p-1) t}\right)^{\frac{1}{(p-1)}}\right| \\
& =\left|\left(\frac{1}{d(t)+(p-1) t}\right)^{\frac{1}{(p-1)}}-\left(\frac{1}{(p-1) t}\right)^{\frac{1}{(p-1)}}\right| \\
& =\left|\left(\frac{1}{(p-1) t}\right)^{\frac{1}{(p-1)}}\left(\frac{1}{\frac{d(t)}{(p-1) t}+1}\right)^{\frac{1}{(p-1)}}-\left(\frac{1}{(p-1) t}\right)^{\frac{1}{(p-1)}}\right| \\
& =\left(\frac{1}{(p-1) t}\right)^{\frac{1}{(p-1)}}\left|\left(\frac{d(t)}{(p-1) t}+1\right)^{-\frac{1}{(p-1)}}-1\right| \\
& =\left(\frac{1}{(p-1) t}\right)^{\frac{1}{(p-1)}}\left(1-\left(\frac{d(t)}{(p-1) t}+1\right)^{-\frac{1}{(p-1)}}\right) .
\end{aligned}
$$

Since $\left|\frac{d(t)}{(p-1) t}\right|<1$ for $t$ large enough. Let $\eta(t)=\left(1+\frac{d(t)}{(p-1) t}\right)^{-\frac{1}{(p-1)}}$, by the mean value Theorem and if we suppose that $\left|\frac{d(t)}{(p-1) t}\right| \leq \frac{1}{2}$, we obtain

$$
\left|\eta^{\prime}(t)\right| \leq \frac{1}{p-1} \times 2^{1+\frac{1}{p-1}}
$$


Therefore

$$
\left|1-\left(1+\frac{d(t)}{(p-1) t}\right)^{-\frac{1}{(p-1)}}\right| \leq \frac{1}{p-1} \times 2^{1+\frac{1}{p-1}}\left|\frac{d(t)}{(p-1) t}\right|
$$

As we have seen above, $d(t)$ is bounded by $M_{1}$ then we conclude

$$
\left|\xi(t)-\left(\frac{1}{(p-1) t}\right)^{\frac{1}{(p-1)}}\right| \leq C t^{-1-\frac{1}{p-1}}
$$

With $C=\left(\frac{1}{p-1}\right)^{\frac{p}{p-1}} \times 2^{1+\frac{1}{p-1}} M_{1}$.

We recall that $z$ has a constant sign on $\left[T_{0},+\infty[\right.$ and $z$ and $u$ have the same sign. As in Section 4, we set $u=v+z$ and $\psi=u+w$, we distinguish two cases

- If $z>0$, this implies that for $t$ large enough $u>0$ and $|\psi|=\psi$. Then

$$
|| \psi\left|-\left(\frac{1}{(p-1) t}\right)^{\frac{1}{(p-1)}}\right| \leq\left|u-\left(\frac{1}{(p-1) t}\right)^{\frac{1}{(p-1)}}\right|+|w| \leq K t^{-1-\frac{1}{p-1}} .
$$

Indeed,

$$
\begin{aligned}
\left|u-\left(\frac{1}{(p-1) t}\right)^{\frac{1}{(p-1)}}\right| & \leq|u-z|+|z-\xi|+\left|\xi-\left(\frac{1}{(p-1) t}\right)^{\frac{1}{(p-1)}}\right| \\
& \leq M e^{-\lambda_{2} t}+k e^{-\delta t}+C t^{-1-\frac{1}{p-1}}
\end{aligned}
$$

Since we have (5.7), we obtain (1.9).

- If we suppose that $z<0$, then implies that $u<0$. By similar calculations we obtain the same result.

Indeed, $|\psi|=-\psi$, and we have 


$$
\begin{aligned}
|| \psi\left|-\left(\frac{1}{(p-1) t}\right)^{\frac{1}{(p-1)}}\right| & \leq\left|-u-\left(\frac{1}{(p-1) t}\right)^{\frac{1}{(p-1)}}\right|+|w| \\
& \leq K t^{-1-\frac{1}{p-1}}
\end{aligned}
$$

since

$$
\begin{aligned}
\left|-u-\left(\frac{1}{(p-1) t}\right)^{\frac{1}{(p-1)}}\right| & \leq|u-z|+|-z-\xi|+\left|\xi-\left(\frac{1}{(p-1) t}\right)^{\frac{1}{(p-1)}}\right| \\
& \leq M e^{-\lambda_{2} t}+k e^{-\delta t}+C t^{-1-\frac{1}{p-1}} .
\end{aligned}
$$

Also since we have (5.7), finally we obtain (1.9).

\section{Proof of Proposition 1.3}

(i) If $\psi$ is an odd function, then $\psi(0,-x)=-\psi(0, x)$. It implies for all $t>0, \quad \psi(t,-x)=-\psi(t, x)$.

In that case

$$
u(t)=P \psi(t, x)=\frac{1}{|\Omega|} \int_{\Omega} \psi(t, x) d x=0 .
$$

Moreover, we know that $\psi=u+w$, where $w(t) \in N^{\perp}$ for all $t \geq 0$ and (cf. Proposition 3.1) we have

$$
\|w(t)\|_{L^{\infty}(\Omega)} \leq K e^{-\lambda_{2} t},
$$

hence the solution $\psi$ satisfies (1.8).

(ii) If $\psi(0, x) \geq 0$ and $\psi$ does not vanish a.e in $\Omega$, then for all $t \geq 0$ $\psi(t, x)>0$, it implies that

$$
\int_{\Omega} \psi(t, x) d x>0
$$


We suppose that we have $\|\psi(t, \cdot)\|_{\infty} \leq C e^{-\lambda_{2} t}$ and we consider the problem (1.1) then we integrate on $\Omega$, we obtain

$$
\int_{\Omega} \psi_{t}(t, x) d x=-\int_{\Omega} g(\psi(t, x)) d x .
$$

An elementary calculation shows that we have

$$
\begin{aligned}
\int_{\Omega} g(\psi(t, x)) d x & \leq \frac{c}{p} \int_{\Omega}|\psi(t, x)|^{p} d x \\
& \leq \frac{c}{p} \int_{\Omega}|\psi(t, x)|^{p-1} \psi(t, x) d x \\
& \leq \frac{c}{p} \int_{\Omega}\|\psi(t, x)\|_{\infty}^{p-1} \psi(t, x) d x \\
& \leq \frac{c}{p} C^{p-1} e^{-(p-1) \lambda_{2} t} \int_{\Omega} \psi(t, x) d x
\end{aligned}
$$

Now we set $y(t)=\int_{\Omega} \psi(t, x) d x$. From (6.2), we deduce

$$
y^{\prime}(t) \geq-M e^{-\delta t} y(t)
$$

with $M=\frac{c}{p} C^{p-1}$ and $\delta=(p-1) \lambda_{2}$.

Since $y(t)>0$ by $(6.1)$, we can integrate in the interval $[0, t]$, we obtain

$$
y(t) \geq y(0) \exp \left\{-M \int_{0}^{t} e^{-\delta s} d s\right\} \geq y(0) \exp \left\{-\frac{M}{\delta}\right\}>0 .
$$

Hence $y$ does not tend to 0 for $t$ large, this contradicts our hypothesis and we conclude that $y$ satisfies (1.7).

\section{References}

[1] P. Bénilan, H. Brézis, Solutions faibles d'équations d'évolution dans les espaces de Hilbert, Annales de l'institut Fourier, 22 no. 2 (1972), p. 311-329.

[2] H.Brézis, Opérateurs maximaux monotones et semi-groupes de contractions dans les espaces de Hilbert.

[3] C. M.Dafermos, Asymptotic behavior of solutions of evolution equations, Nonlinear Evolution Equations, M. G. Crandall Ed, Academic Press, New-York 1978, 103-123. 
[4] A. Haraux, Slow and fast decay of solutions to some second order evolution equations, J. Analyse Mathématiques. 95 (2005), 297-321.

[5] A. Haraux, Decay rate of the range component of solutions to some semilinear evolution equations, J. Analyse Mathématiques.13 (2006), 435445.

[6] A. Haraux, M. A. Jendoubi and O. Kavian, Rate of decay to equilibrium in some semilinear parabolic equations, Journal of Evolution equations 3 (2003), 463-484.

[7] A. Haraux, Systèmes dynamiques dissipatifs et applications, Collection R. M. A. 17, Collection dirigé par P. G. Ciarlet et J. L. Lions, Masson, Paris 1991.

[8] A. Haraux, M. Kirane, Estimations $C^{1}$ pour des problèmes paraboliques semi-linéaires, Annales de la faculté des sciences de Toulouse Sér. 5, 5 no. 3-4 (1983), p. 265-280. 